\title{
C-08
}

\section{BAJA PRESIÓN EN ASPERSORES DE IMPACTO EN COBERTURAS DE RIEGO: ANÁLISIS TÉCNICO Y AGRONÓMICO}

\author{
Robles, O. $^{1}$ y Zapata, $N^{2}$ \\ ${ }^{1}$ Personal Investigador en Formación. Depto. Suelo y Agua, Estación Experimental Aula Dei \\ (EEAD-CSIC). Avda. Montañana 1005, 50059, Zaragoza, España, E-mail: \\ coctaviorobles@eead.csic.es \\ ${ }^{2}$ Científico Titular, Depto. Suelo y Agua, Estación Experimental Aula Dei (EEAD-CSIC). \\ Avda. Montañana 1005, 50059, Zaragoza, España, E-mail: v.zapata@csic.es
}

\section{Resumen}

La eficiencia energética en riego presurizado se ha abordado desde la gestión hidráulica de las infraestructuras de red y la eficiencia en los bombeos, sin embargo, es necesario analizar los ahorros que pueden realizarse a escala de parcela. Resultados preliminares indican que la reducción de la presión de funcionamiento de un aspersor de impacto de $300 \mathrm{kPa}$ a $200 \mathrm{kPa}$ ligeramente reduce la uniformidad del riego. En este trabajo se evalúa en campo la calidad del riego y los resultados agronómicos de tres configuraciones aspersor+presión: 1) un aspersor de impacto convencional trabajando a 300 $\mathrm{kPa}(\mathrm{AC} 300), 2)$ un aspersor convencional trabajando a $200 \mathrm{kPa}$ (AC 200) y 3) un aspersor modificado con una pequeña placa deflectora sobre el brazo de impacto trabajando a 200 $\mathrm{kPa}$ (APD 200). En una parcela experimental de cobertura total formada por 12 subparcelas, se realizó un ensayo en bloques al azar sobre un cultivo de maíz durante la campaña 2015. Se caracterizaron las condiciones meteorológicas (velocidad y dirección del viento) de los riegos aplicados al maíz. Para cada tratamiento se realizaron ensayos de uniformidad, evaluaciones agronómicas, cosecha manual de biomasa aérea y cosecha de grano. Los resultados indican que la uniformidad media evaluada no fue significativamente diferente entre tratamientos. La biomasa y producción de grano del tratamiento de $300 \mathrm{kPa}$ resultaron menores que las de los tratamientos a $200 \mathrm{kPa}$ (AC 200 y APD 200), no resultando significativas las diferencias entre ambos tratamientos de $200 \mathrm{kPa}$. Se detectó un problema en el crecimiento de cultivo que afectó principalmente a las subparcelas de $300 \mathrm{kPa}$, por lo que los resultados deben de considerarse con cuidado y realizar el ensayo en una o dos temporadas más.

\section{1- Introducción}

La expansión de los sistemas de riego presurizados ha tenido gran importancia en los últimos años debido a los Planes de Modernización de regadíos impulsados por el Ministerio de Agricultura. El uso de sistemas de riego por aspersión genera incrementos en la productividad del agua al modernizar una zona de riego, pero los costes energéticos implican un incremento muy importante en los costes de producción. Debido al importante aumento del consumo energético en el regadío, al coste de la amortización de las infraestructuras modernizadas y al elevado coste de la energía eléctrica, los beneficios de los agricultores se han visto reducidos de forma drástica, peligrando la viabilidad de las explotaciones recién modernizadas.

Conscientes de lo anterior, diversos trabajos se han realizado entorno a la eficiencia del uso del agua mediante la reducción de las pérdidas (Playán et al, 2005; Cavero et al, 2008).También se ha abordado en la literatura la mejora de la eficiencia energética desde 
diferentes perspectivas como la gestión de infraestructuras de red general de riego (CarrilloCobo et al, 2010) y las condiciones óptimas de operación de las estaciones de bombeo (Moreno et al, 2010; Lamaddalena y Khila, 2012). Sin embargo, en necesario seguir avanzando en la optimización energética de todo el sistema, prestando atención también al riego en parcela.

Las zonas de riego por aspersión para cultivos extensivos se han diseñado para proporcionar una presión en la boquilla del aspersor de impacto de entre $300 \mathrm{kPa}$ a $350 \mathrm{kPa}$. Según varios autores (Keller y Bliesner, 1990; Tarjuelo, 2005) la calidad de riego (uniformidad y eficiencia) depende de diversos factores, tales como: las características del aspersor, el tamaño y forma de las boquillas, la presión de trabajo y su variabilidad, la altura de aspersor, el espaciamiento entre ellos y las condiciones meteorológicas durante el riego. Sin embargo, en un trabajo reciente Playán et al, (2006) presentaron ensayos de uniformidad de riego de dos modelos de aspersor de impacto trabajando a diferentes presiones $(200,300$ y $400 \mathrm{kPa}$ ) sus resultados apuntan a que las diferencias en uniformidad entre 200 y $300 \mathrm{kPa}$ no son muy relevantes. La reducción de la presión en boquilla a 200 $\mathrm{kPa}$ puede suponer un ahorro considerable de energía, sin embargo tenemos que asegurarnos que la calidad del riego cumple unos mínimos y que la producción del cultivo no se ve afectada.

Un riego a baja presión tiene otros efectos como la disminución del área efectiva de riego y la generación de gotas grandes que poseen mayor resistencia al ser arrastradas. También hay que citar el posible efecto que la distribución de tamaños de gotas tiene sobre los procesos erosión-sellado del suelo (Seginer, 1965; Kohl et al, 1985) y, por ende, los de infiltración (Mohammed y Kohl, 1987). Las técnicas de no laboreo o de laboreo de conservación pueden mitigar este efecto negativo sobre el suelo.

El objetivo de este trabajo es caracterizar experimentalmente mediante un ensayo de campo las diferencias en calidad del riego y producción de un cultivo entre un riego por aspersión a $300 \mathrm{kPa}$ y a $200 \mathrm{kPa}$. Para ello se analizarán tres configuraciones aspersor+presión que comprenden: 1) un aspersor de impacto convencional trabajando a $300 \mathrm{kPa}$ (AC 300), 2) un aspersor convencional trabajando a $200 \mathrm{kPa}$ (AC 200) y 3) un aspersor modificado con una pequeña placa deflectora sobre el brazo de impacto trabajando a $200 \mathrm{kPa}$ (APD 200).

\section{2- Materiales y métodos}

El ensayo se realizó en una finca experimental de la Estación Experimental Aula Dei (EEAD-CSIC) en Montañana Zaragoza. El clima de la zona es mediterráneo semiárido con precipitación y evapotranspiración media anual de $322 \mathrm{~mm}$ y $1100 \mathrm{~mm}$, respectivamente, las temperaturas promedio máximas y mínimas oscilan entre $20,9^{\circ} \mathrm{C}$ y $8,5^{\circ} \mathrm{C}$. El suelo de la zona se clasifica como xerofluvente típico.

La parcela experimental tiene una superficie de 1,8 ha, dividida en 12 subparcelas elementales de $324 \mathrm{~m}^{2}$. Se ensayan tres configuraciones aspersor+presión: 1) un aspersor de impacto convencional trabajando a $300 \mathrm{kPa}$ (AC 300), 2) un aspersor convencional trabajando a $200 \mathrm{kPa}(A C 200)$ y 3) un aspersor modificado con una pequeña placa deflectora sobre el brazo de impacto trabajando a $200 \mathrm{kPa}$ (APD 200). Los tratamientos se distribuyeron en bloques al azar con cuatro repeticiones. La configuración de cada una de las 12 subparcelas fue cuadrada de $18 \mathrm{~m} \times 18 \mathrm{~m}$ y los cuatro aspersores que la componen están regulados por una válvula de sector (Figura 1). Los aspersores que operaban a una presión de $200 \mathrm{kPa}$ se equiparon con boquillas de $5,2 \mathrm{~mm}+2,4 \mathrm{~mm}$, mientras que los aspersores que operaban a $300 \mathrm{kPa}$ estaban equipados con boquillas de $4,4 \mathrm{~mm}+2,4 \mathrm{~mm}$. Estas diferencias en tamaños de boquilla compensan las diferencias en presión, de forma que la pluviometría media es la misma. La presión en cada una de las 12 subparcelas se mantuvo constante de acuerdo a su tratamiento correspondiente (200 kPa o $300 \mathrm{kPa})$ con válvulas reguladoras de presión instaladas en cada una de las válvulas de sector. La presión de funcionamiento se media en continuo (frecuencia de 5 minutos) en cada una de las subparcelas mediante un transductor de presión instalado en uno de los aspersores de cada 
subparcela (Figura 1). Los bordes de la parcela (V1 y V14) fueron regadas independientemente de las 12 subparcelas que componen el ensayo.

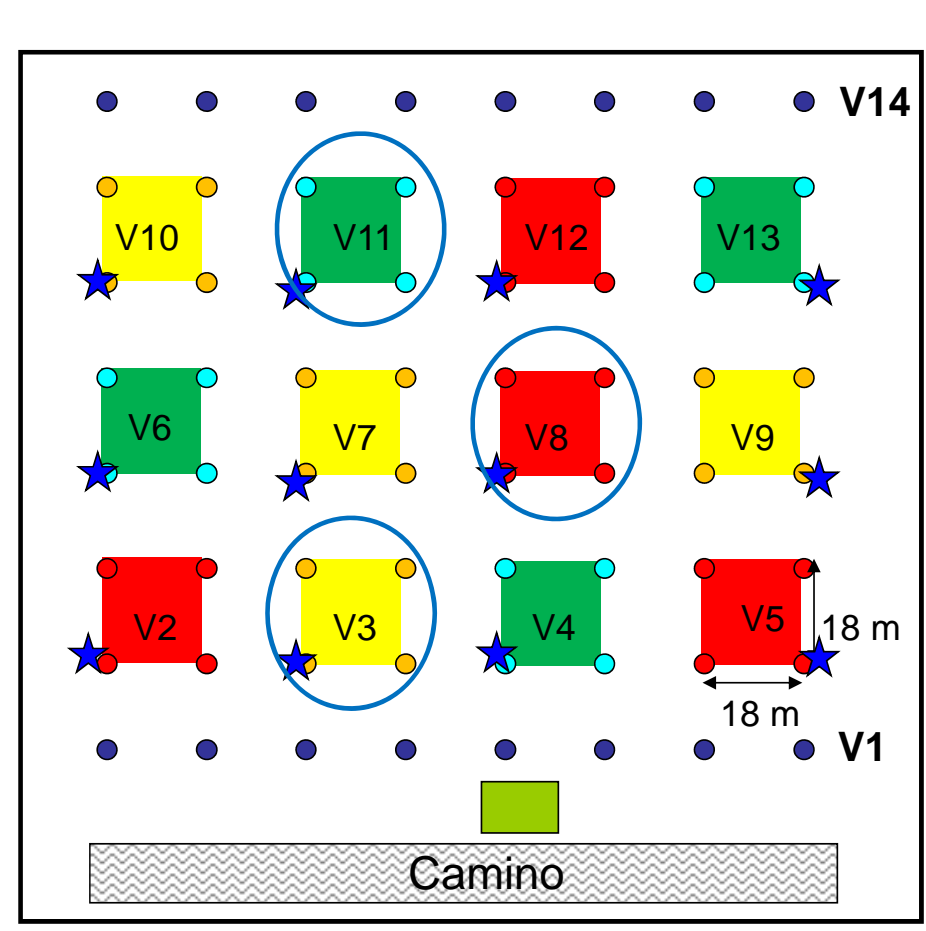

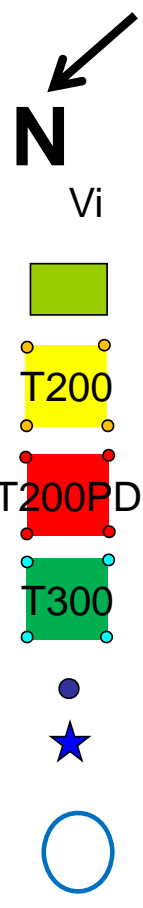

Electroválvulas

Caseta de riego

AC $200 \mathrm{kPa}, 5,2+2,4$

APD 200 kPa, 5,2 + 2,5

AC $300 \mathrm{kPa}, 4,4+2,4$

Aspersores bordadura

Transductores de presión /manómetros

Pluviómetros

Figura 1. Diseño experimental del sistema de riego de cobertura total. Cada experimento analizado se muestra con un color diferente.

En la parcela se sembró maíz (Pioneer P1758) con densidad de 89500 semillas ha ${ }^{-1}$ en abril de 2015, la separación entre filas fue de $0,75 \mathrm{~m}$ y entre plantas de aproximadamente $0,15 \mathrm{~m}$. Se aplicó una fertilización antes de la siembra de $\mathrm{N}\left(64 \mathrm{~kg} \mathrm{ha}^{-1}\right), \mathrm{P}_{2} \mathrm{O}_{5}\left(120 \mathrm{~kg} \mathrm{ha}^{-1}\right)$ y $\mathrm{K}_{2} \mathrm{O}\left(120 \mathrm{~kg} \mathrm{ha}^{-1}\right)$. Además, se aplicó $100 \mathrm{~kg} \mathrm{ha}^{-1}$ de solución $\mathrm{N}-32$ con agua de riego después de la nascencia del maíz. Las hierbas y plagas fueron controladas de acuerdo a las prácticas de la zona (solución CAMIX 3,75 I ha-1 como preemergente y solución Clorpirifós en 2 I ha ${ }^{-1}$ como insecticida).

Tras la nascencia del cultivo se instalaron mallas de 25 pluviómetros en tres subparcelas, una en cada tratamiento (V3, V8 y V11, Figura 1). Se evaluaron 6 de los riegos aplicados al maíz para determinar las uniformidades de riego (Christiansen, 1942) ante diversas condiciones meteorológicas. La duración aproximada del riego en cada ensayo fue de 2 horas como mínimo. Se utilizaron los registros agrometeorológicos de la estación SIAR de Montañana localizada a $1200 \mathrm{~m}$ del ensayo. Se realizó un análisis de la frecuencia de las diferentes intensidades y direcciones del viento durante los eventos de riego. Para ello se clasificó la intensidad del viento de los eventos de riego en tres categorías: viento bajo, medio y alto. El cálculo de las necesidades hídricas netas del cultivo se realizó a partir de la evapotranspiración diaria del cultivo de referencia (Método Penman-Monteith, Allen et al, 1998) facilitado por la estación SIAR antes mencionada y de los coeficientes de cultivo basados en la integral térmica propuesta para el cultivo del maíz en la zona por Martínez-Cob (2008), considerando la precipitación efectiva (75\% de la precipitación real).

Se realizaron dos medidas agronómicas después de la floración del maíz: a) la altura de planta, y b) la radiación fotosintéticamente activa interceptada por el cultivo (IPAR). La altura de planta se midió en 20 puntos internos de cada una de las subparcelas, utilizando una regla de madera con precisión en centímetros. La IPAR se midió en 12 puntos de cada sector, utilizando un ceptómetro (Sunscan Delta-T, Cambridge, UK) para medir la radiación interceptada al nivel del suelo y un sensor de fracción de haz (BF3 Delta-T, Cambridge, UK) 
para medir la radiación solar interceptada fuera de la parcela. IPAR se calculó como la diferencia entre las lecturas del ceptómetro y del sensor de fracción.

Se realizó cosecha manual de un 1,4\% de la superficie de la subparcela para estimar la biomasa aérea. Las plantas se cortaron por encima de la superficie del suelo separando la mazorca del resto de la planta. A su vez, se separó de la mazorca el grano y el zuro. Toda la biomasa aérea (grano, planta y mazorca) fue pesada por separado y posteriormente secada a $60^{\circ} \mathrm{C}$ durante 72 horas, obteniendo el peso de la biomasa seca. Se determinó el índice de cosecha (IC) de cada subparcela como la relación entre el peso del grano al $14 \%$ y la biomasa aérea seca.

La cosecha final se llevó a cabo en septiembre de 2015, una cosechadora comercial recolectó el grano de cada subparcela por separado que se pesó in-situ con ayuda de un remolque pesador (precisión de $1 \mathrm{~kg}$ ). En cada una de las 12 cosechas se aisló una muestra de $1 \mathrm{~kg}$ para verificar la humedad del grano. La producción de grano por subparcela se estimó convirtiendo el peso del grano al 14\% de humedad.

Las variables analizadas en este trabajo: presión de trabajo en aspersor, altura de planta, IPAR, biomasa aérea, IC y producción de grano fueron tratadas estadísticamente con ANOVA al $95 \%$ de confiabilidad $(p=0,05)$, resaltando las diferencias significativas entre tratamientos.

\section{3- Resultados y discusión}

El caudal medio de cada aspersor fue de 1607 I $\mathrm{h}^{-1}$ (APD 200), $1584 \mathrm{I} \mathrm{h}$ (AC 200) y $1597 \mathrm{I} \mathrm{h}^{-1}$ (AC 300), las diferencias entre éstas fueron inferiores a 1,3\%. La pluviometría media de los tres tratamientos fue de $4,9 \mathrm{~mm} \mathrm{~h}^{-1}$. La dosis estacional de riego aplicada al maíz para la campaña de riego 2015 fue de $635 \mathrm{~mm}$ distribuido en 35 riegos. Salvo los riegos de nascencia, la duración de los mismos era de entre 3 y 4 horas (generalmente de las 07:00 a 11:00 hora civil) con una frecuencia de entre uno a tres eventos por semana, en función de las necesidades del cultivo.

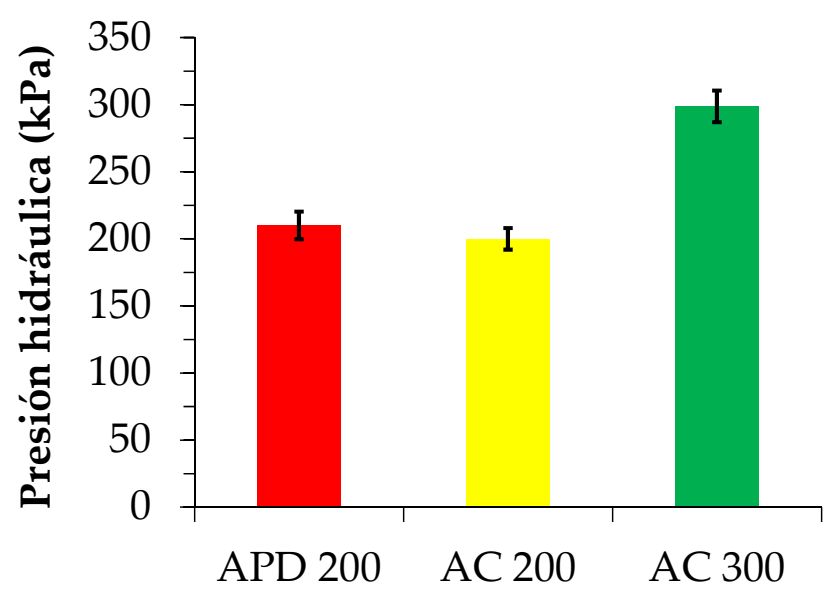

Figura 2. Presiones registradas por los transductores en los aspersores de impacto de cada tratamiento.

El promedio por tratamiento de las presiones registradas por los transductores se muestra en la Figura 2. Los valores fueron de $210 \mathrm{kPa}, 200 \mathrm{kPa}$ y $300 \mathrm{kPa}$ para los tratamientos APD 200, AC 200 y AC 300, respectivamente. En general, para todo el experimento la desviación estándar promedio fue de $10 \mathrm{kPa}$.

El viento promedio durante los 35 eventos de riego aplicados al maíz en la campaña 2015 fue de 2,1 $\mathrm{m} \mathrm{s}^{-1}$. La dirección del viento predominante fue OSO en 22\% (8 riegos) de los casos (Figura 3a), ESE en el 15\% (5 riegos) y en el resto de los eventos la dirección fue cambiante. En alrededor de 14\% de los casos (5 riegos) se presentó un viento superior a los 
$4 \mathrm{~m} \mathrm{~s}^{-1}$ ocurriendo en los meses de junio, julio y agosto (Figura 3b). En toda la campaña se presentaron vientos menores a $2 \mathrm{~m} \mathrm{~s}^{-1}$ en 18 eventos de riego (51\% de los casos).
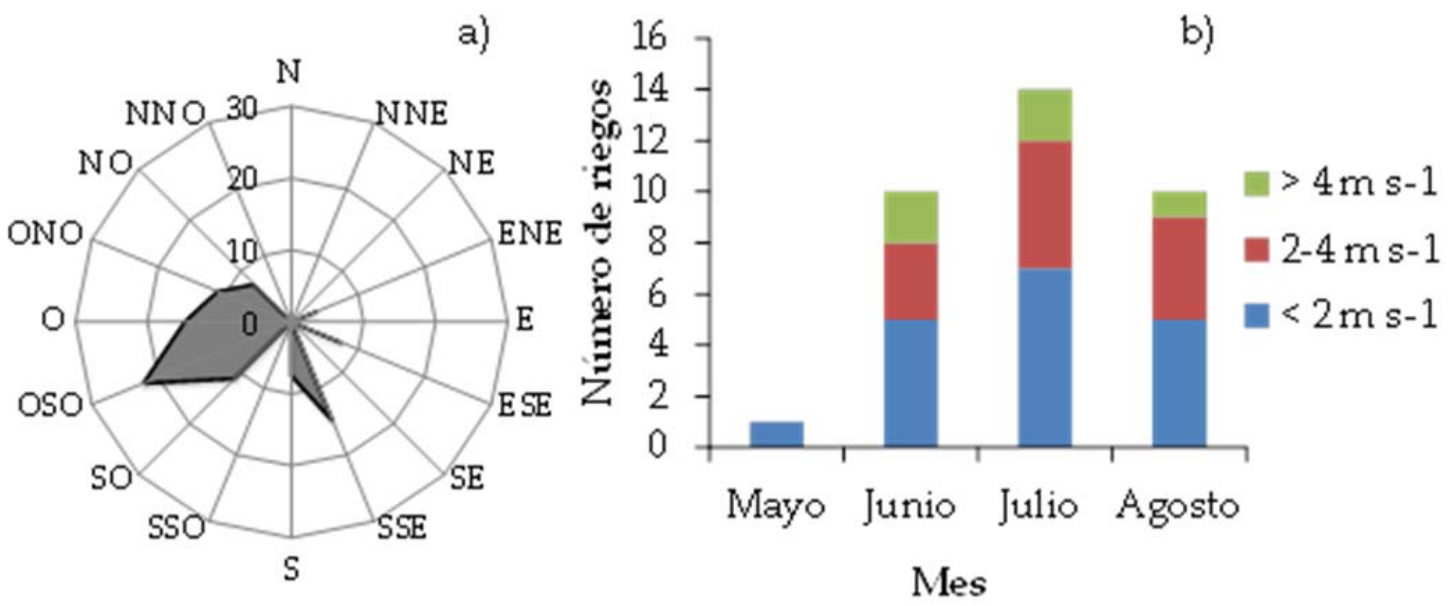

Figura 3. Comportamiento del viento en los eventos de riego de la campaña del maíz. a) frecuencia de dirección de viento durante los riego de maíz y b) frecuencia de velocidades baja, media y alta $\left(<2 \mathrm{~m} \mathrm{~s}^{-1}, 2-4 \mathrm{~m} \mathrm{~s}^{-1} y>4 \mathrm{~m} \mathrm{~s}^{-1}\right)$ durante los meses en los que ocurrió el riego.

El CU promedio de los seis riegos evaluados fue de $84 \%$, $84 \%$ y $86 \%$, para los tratamientos APD 200, AC 200 y AC 300, respectivamente. Cuatro de los riegos evaluados se efectuaron en condiciones de viento en calma $\left(<2 \mathrm{~m} \mathrm{~s}^{-1}\right)$, uno con viento medio $(>=$ $2 \mathrm{~m} \mathrm{~s}^{-1} \mathrm{y}<4 \mathrm{~m} \mathrm{~s}^{-1}$ ) y otro con viento alto $\left(>4 \mathrm{~m} \mathrm{~s}^{-1}\right)$. La Figura 4 muestra dos de los riegos evaluados en dos casos de viento muy diferente, viento bajo $\left(1 \mathrm{~m} \mathrm{~s}^{-1}\right)$ y viento alto $\left(4,5 \mathrm{~m} \mathrm{~s}^{-1}\right)$. En ambos caso el tiempo de riego fue de 2 horas. En el riego efectuado en condiciones de viento en calma, el CU fue mayor para el tratamiento AC 300 (91\%), seguido del tratamiento APD 200 (89\%), siendo el tratamiento de menor CU (88\%) el tratamiento AC 200. Las diferencias son pequeñas y las uniformidades medias de todos los tratamientos están por encima del mínimo CU recomendado. En cuanto al riego efectuado en condiciones de viento alto, la mayor uniformidad se obtuvo en el tratamiento AC 200 (77\%), siendo el menor valor el del tratamiento APD 200 (66\%). En este caso todos los valores están por debajo del CU recomendado. Los valores medios del CU de los seis riegos evaluados no indican una relación clara entre tratamientos, pues las diferencias no son estadísticamente significativas entre ninguno de ellos. 


\section{APD 200 kPa}

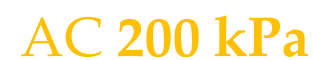

$\mathrm{AC} 300 \mathrm{kPa}$
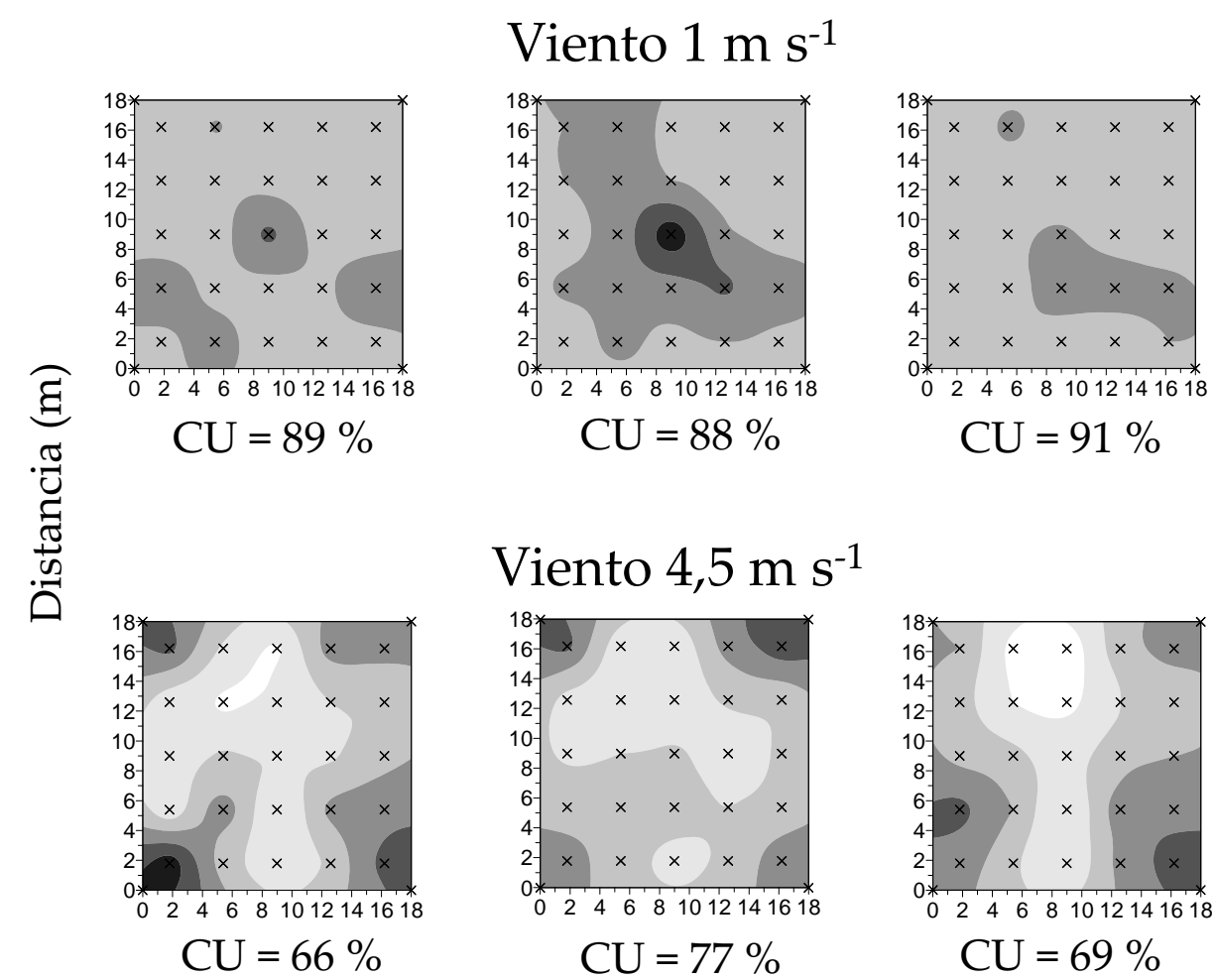

Viento $4,5 \mathrm{~m} \mathrm{~s}^{-1}$
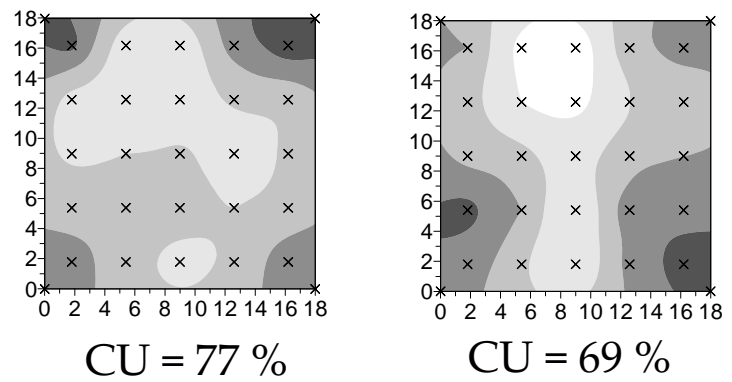

Distancia (m)

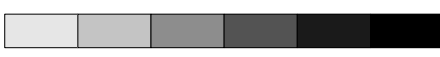

\section{$2,5 \quad 4 \quad 5,5 \quad 7 \quad 8,5 \quad 10(\mathrm{~mm})$}

Figura 4. Coeficientes de uniformidad (CU) y mapas de distribución de agua en una de las subparcelas de cada tratamiento en dos eventos de la campaña de riego, uno de viento bajo y otro de viento alto. La escala de pluviometría se muestra en grises con intervalos de 1,5 $\mathrm{mm}$.

Los valores medios por tratamiento de la altura de planta se presentan en la Figura 5a. El análisis de los datos nos indica que la altura de planta en los tratamientos de $200 \mathrm{kPa}$, APD 200 y AC 200, no presenta diferencias estadísticamente significativas. Tampoco es significativa la diferencia en altura de planta entre los tratamientos AC 200 y AC 300. Sin embargo, la diferencia es significativa entre los tratamientos APD 200 y AC 300. Hay que comentar que en el tratamiento $A C 300$, en dos de sus repeticiones, las localizadas en la parte final de la parcela (válvulas 11 y 13 de la Figura 1), la altura de planta resultó claramente por debajo de la media global. En esta parte se observó visualmente retraso en la fase de nascencia en relación con el resto de subparcelas.

Las medidas de IPAR (Figura 5b) no presentaron diferencias significativas entre tratamientos. En general no se aprecia una relación clara entre la radiación interceptada por el cultivo y la altura del mismo. 


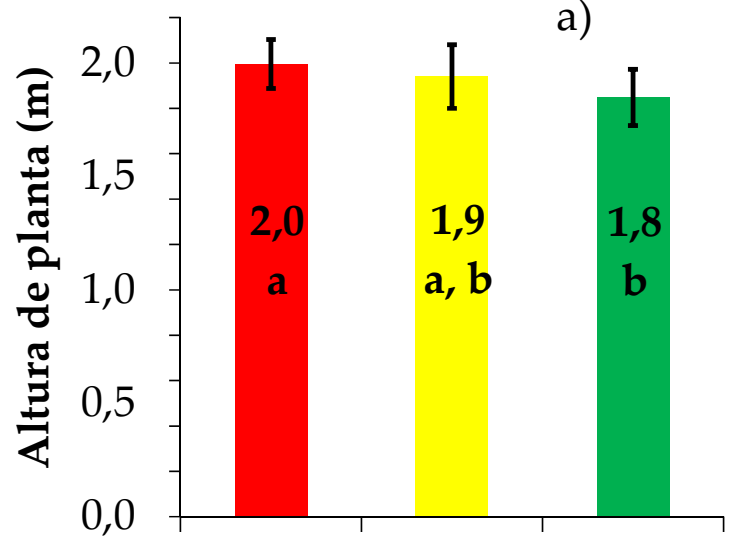

APD 200 AC 200 AC 300

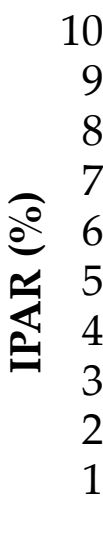

00
90
80
70
60
50
40
30
20
10
0 b)
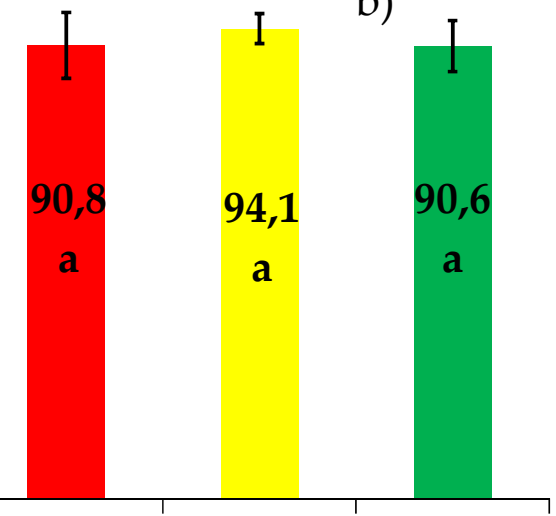

APD 200 AC $200 \quad$ AC300

Figura 5. Evaluaciones agronómicas sobre el maíz. a) altura de planta promedio ( $m$ ) y b) radiación solar interceptada por el cultivo (\%). Los valores de ambas imágenes se expresan para cada tratamiento analizado (APD 200, AC 200 y AC 300). Los números seguidos de letras distintas presentan diferencias significativas al $95 \%$ de confiabilidad $(p=0,05)$ para cada variable.

La Tabla 1 muestra la biomasa aérea, la producción en grano, la eficiencia en el uso del agua y el índice de cosecha de cada una de las subparcelas del ensayo. La menor biomasa y menor producción de grano se dio en la válvula 13 (V13, tratamiento AC 300) con 22,1 Ton ha ${ }^{-1}$ y 13,2 Ton ha ${ }^{-1}$, respectivamente. Además, la V13, obtuvo la menor cantidad de grano por metro cúbico de agua (menor eficiencia en el uso del agua). Sin embargo, fue la que obtuvo mayor producción de grano por biomasa recolectada (mayor IC). También fue la V13 la que menor altura de planta presentó. Los dos valores mayores de producción en grano se dieron en las válvulas V5 y V2, ambas del mismo tratamiento, APD 200. En estas mismas válvulas también se dieron los valores máximos de eficiencia en el uso del agua de todo el ensayo. La V7 (AC 200) fue la que presentó el mayor valor de biomasa aérea.

La producción media del ensayo fue de $14885 \mathrm{~kg} \mathrm{ha}^{-1}$. En promedio se necesitaron $2,34 \mathrm{~m}^{3}$ de agua para producir un kilogramo de grano de maíz. El índice de cosecha medio indica que la producción del grano representa el $52 \%$ de la biomasa recolectada.

Tabla 1. Resultados de la cosecha del maíz. La biomasa aérea, producción del grano al $14 \%$ de humedad y el uso eficiente del agua se muestran para cada subparcela del experimento.

\begin{tabular}{|c|c|c|c|c|c|}
\hline Válvula Tratamiento & $\begin{array}{c}\text { Biomasa aérea } \\
\left(\mathrm{kg} \mathrm{ha}^{-1}\right)\end{array}$ & $\begin{array}{c}\text { Producción de } \\
\text { grano al 14\% } \\
\left(\mathrm{kg} \mathrm{ha}^{-1}\right)\end{array}$ & $\begin{array}{c}\text { Eficiente del uso } \\
\text { del agua }\left(\mathrm{kg} \mathrm{m}^{-3}\right)\end{array}$ & $\begin{array}{c}\text { IC } \\
(-)\end{array}$ \\
\hline 2 & APD 200 & 28941 & 16151 & 2,54 & 0,48 \\
\hline 3 & AC 200 & 28845 & 15234 & 2.40 & 0,48 \\
\hline 4 & AC 300 & 29821 & 15115 & 2,38 & 0,47 \\
\hline 5 & APD 200 & 28224 & 16286 & 2,56 & 0,52 \\
\hline 6 & AC 300 & 24335 & 14093 & 2,22 & 0,54 \\
\hline 7 & AC 200 & 30200 & 15029 & 2,37 & 0,53 \\
\hline 8 & APD 200 & 29547 & 15485 & 2,44 & 0,50 \\
\hline 9 & AC 200 & 25782 & 15242 & 2,40 & 0,54 \\
\hline 10 & AC 200 & 27582 & 14665 & 2,31 & 0,53 \\
\hline
\end{tabular}




\begin{tabular}{|l|l|l|l|l|l|}
\hline 11 & AC 300 & 22248 & 13468 & 2,12 & 0,54 \\
\hline 12 & APD200 & 23285 & 14702 & 2,32 & 0,53 \\
\hline 13 & AC 300 & 22097 & 13148 & 2,07 & 0,55 \\
\hline
\end{tabular}

Las medias por tratamiento de biomasa aérea y producción de grano al $14 \%$ de humedad se muestran en la Figura 6. La biomasa aérea no fue significativamente diferente entre los tres tratamientos analizados. En cuanto a la producción en grano, los análisis estadísticos indican que entre los dos tratamientos de baja presión (AC 200 y APD 200) no hubo diferencias significativas. Sin embargo, sí que hubo diferencias entre los tratamientos de $300 \mathrm{kPa}$ y los de $200 \mathrm{kPa}$, resultando el tratamiento de presión estándar (300 kPa) el que menor producción presentó. Estos resultados son llamativos, pues se esperaba que el tratamiento de presión estándar (AC 300) resultase en mayor o igual producción que los tratamientos de baja presión (AC 200 y APD 200).

Se hizo un análisis pormenorizado de las mediciones del ensayo y se constató que durante la fase de nascencia las subparcelas de las válvulas V10, V11 y V13, sufrieron un retraso comparadas con el resto. Este retraso afectó al desarrollo de la planta como lo indican las medidas de altura de planta y las de producción en grano. Se intentó aislar este problema realizando de nuevo el análisis estadístico ANOVA descartando las repeticiones en las que se observaron problemas. Los resultados del nuevo análisis indicaron que no había diferencias estadísticamente significativas entre los tratamientos AC 200 y APD 200 (igual que en el análisis anterior); tampoco entre los tratamientos AC 200 y AC 300, pero sí que las había entre los tratamientos AC 300 y APD 200, con menor producción en grano para el tratamiento AC 300. A pesar de que las diferencias se suavizaban, la producción en grano del tratamiento estándar seguía siendo menor. También se realizaron una serie de comprobaciones sobre el suelo de los diferentes tratamientos, así se comparó el contenido de agua en los primeros $60 \mathrm{~cm}$ del suelo antes de realizar el ensayo agronómico. También se determinaron las diferencias en humedad del suelo por tratamiento tras el ensayo agronómico y en ninguna de las determinaciones se identificaron diferencias significativas. Tampoco se observaron diferencias entre tratamientos en el contenido de nitrógeno en el suelo analizado tras la cosecha. Sin embargo, la revisión del historial de la parcela nos indicó que precisamente en las válvulas donde se localizaban las cuatro repeticiones del tratamiento AC 300, se localizaron los dos tratamientos de menor dosis de riego de un ensayo anterior sobre diferentes dosis de riego en alfalfa. Este hecho nos ha llevado a reorganizar la localización de las repeticiones de los tratamientos y el próximo año de ensayo los tratamientos se volverán a sortear entre las parcelas experimentales. Hemos de decir en nuestra defensa que las producciones medias en grano obtenidas en los tratamientos de baja presión (ADP 200 y AC 200) son comparables con el rendimiento de obtenido en una parcela aledaña en la que se ensaya la misma variedad de maíz regado a $300 \mathrm{kPa}$ (J. Cavero comunicación personal). 


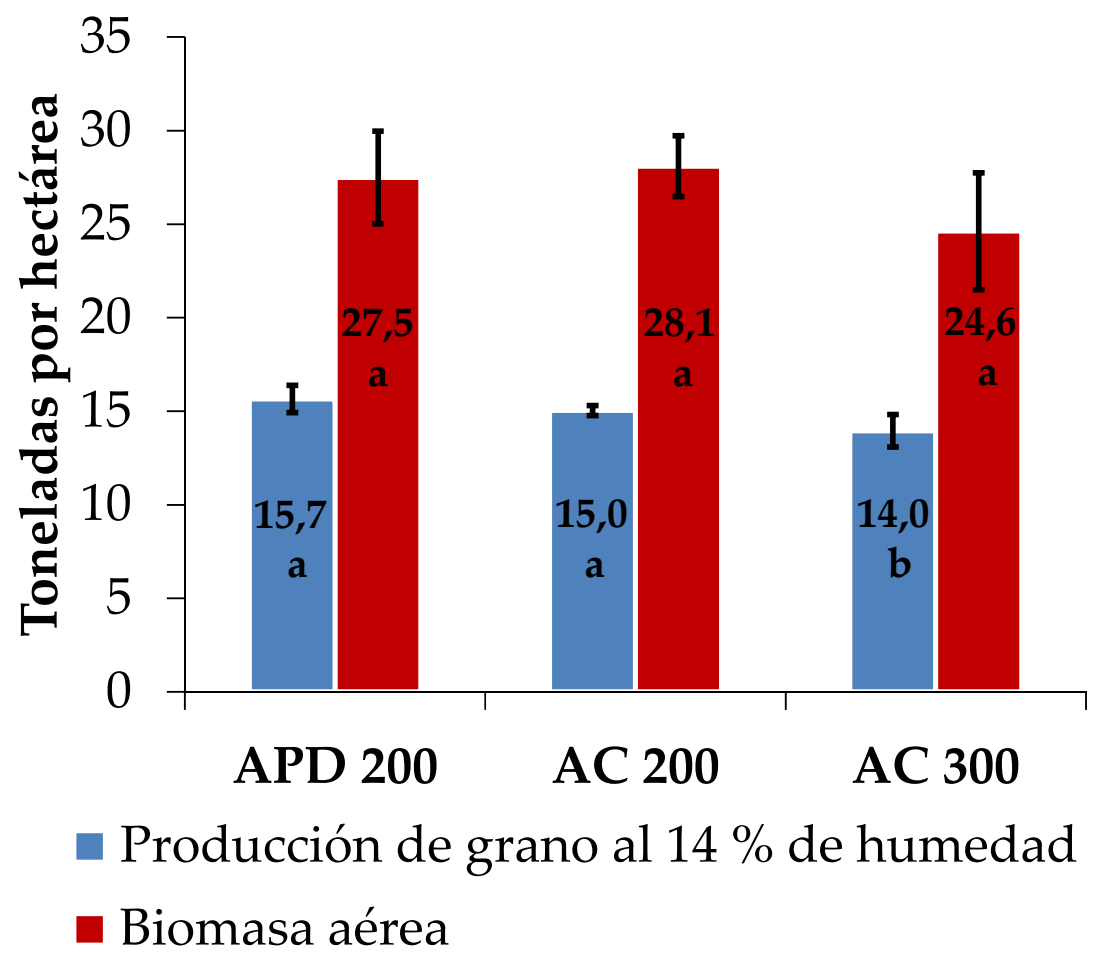

Figura 6. Resultados globales de la cosecha. Se presenta en color distinto la producción de grano al $14 \%$ de humedad y la biomasa aérea obtenida para cada tratamiento del ensayo. Los números seguidos de letras distintas presentan diferencias significativas al $95 \%$ de confiabilidad $(p=0,05)$ para cada variable.

\section{4- Conclusiones y recomendaciones}

Los resultados de los ensayos nos indican que la calidad del riego de las evaluaciones de los riegos realizadas no fue diferente entre los diferentes tratamientos ensayados, APD 200, AC 200 y AC 300. En algunos riegos la uniformidad fue superior para los tratamientos de baja presión y en otros lo contrario. Sin embargo, es necesario analizar la campaña completa de riegos para sacar conclusiones sobre el CU entre tratamientos. En cuanto a la cosecha, los resultados indican una mayor producción de maíz en los tratamientos de $200 \mathrm{kPa}$ respecto a los de presión convencional de $300 \mathrm{kPa}$. En las subparcelas experimentales de la parte final de la parcela (válvulas 10, 11, 12 y 13) se registraron problemas de nascencia que tuvieron su repercusión en la altura de planta, en la biomasa aérea y en la producción de grano. Dado que en esta zona se localizan dos de las repeticiones del tratamiento AC 300, es necesario tomar estos datos con precaución y repetir el ensayo una o dos campañas de cultivos más.

\section{5- Agradecimientos}

La ejecución de esta investigación se respalda por una beca del MICINN del Gobierno de España (AGL2013-48728-C2-1-R). En este trabajo fue posible gracias al apoyo del personal del EEAD-CSIC y CITA-DGA: José Cavero, Eva Medina, Pilar Paniagua, Miguel Izquierdo, Miguel Á. Millán, Vicente Villarroya, César Romano, Pablo García y Laura Pérez.

\section{6- Referencias}

Allen, R.G., Pereira, L.S., Raes, D., Smith, M. (1998). Crop evapotranspiration: Guidelines for computing crop water requirements. Irrigation and Drainage Paper 56. FAO, Rome. 
Carrillo-Cobo, M.T., Rodríguez-Díaz, J.A, Camacho-Poyato (2010). The role of energy audits in irrigated areas. The case of 'Fuente Palmera' irrigation district (Spain). Spain. J. Agric. Res, 8 (S2), 152-161.

Cavero, J., Jiménez, L., Puig, M., Faci, J.M., Martínez-Cob, A. (2008). Maize growth and yield under daytime and nighttime solid-set sprinkler irrigation. Agron. J., 100 (6), 5731579.

Christiansen, J.E. (1942). Irrigation by sprinkling. Agric. Exp. Stn. Bull. 670. Univ. of California, Berkeley.

Keller, J. y Bliesner, R.D. (1990). Sprinkle and trickle irrigation. Van Nostrand Reinhold, New York, NY, 86-89.

Kohl, R.A., DeBoer, D.W., Evenson, P.D. (1985). Kinetic Energy of low pressure spray sprinklers. Trans ASAE, 28 (5), 1526-1529.

Lamaddalena, N. y Khila, S. (2012). Energy saving with variable speed pumps in on-demand irrigation systems. Irrig. Sci., 30, 157-166.

Martínez-Cob, A. (2008). Use of thermal units to estimate corn crop coefficients under semiarid climatic conditions. Irrig. Sci., 26(4), 335-345.

Mohammed, D. y Kohl, R.A. (1987). Infiltration response to kinetic energy. Trans. ASAE, 30(1), 108-111.

Moreno, M.A., Córcoles, J.I., Tarjuelo, J.M., Ortega, J.F. (2010). Energy efficiency of pressurised irrigation networks managed on-demand and under a rotation schedule. Biosyst. Eng., 107 (4), 349-363.

Playán, E., Salvador, R., Faci, J.M., Zapata, N., Martínez-Cob, A., Sánchez, I. (2005). Day and night wind drift and evaporation losses in sprinkler solid-sets and moving laterals. Agr. Water Manage., 76 (3), 139-159.

Playán, E., Zapata, N., Faci, J. M., Tolosa, D., Lacueva, J. L., Pelegrín, J., Salvador, R., Sánchez, I., Lafita, A. (2006). Assessing sprinkler irrigation uniformity using a ballistic simulation model. Agr. Water Manage., 84, 89-100.

Seginer, I. (1965). Tangential velocity of sprinkler drop. Trans. ASAE, 90-93.

Tarjuelo, J.M. (2005). El riego por aspersión y su tecnología. S.A. Mundi-Prensa Libros, España. 